\title{
Reactive power/voltage control in a distribution substation using dynamic programming
}

F.-C.Lu

Y.-Y.Hsu

\begin{abstract}
The reactive power/voltage control problem in a distribution substation is investigated. The purpose is to properly dispatch the shunt capacitors and onload tap changers at the distribution substation based on the forecast hourly loads of a main transformer and its primary bus voltage such that the reactive power flows through the main transformer, and the transformer secondary bus voltage deviations from the desired values can be minimised. Practical constraints on secondary bus voltage limits and the maximum allowable number of switching operations in a day for the onload tap changers are taken into account. An approach based on dynamic programming is presented to reach the desired dispatching schedule. To demonstrate the usefulness of the proposed approach, reactive power/voltage control in a distribution substation within the service area of Taipei City District Office of Taiwan Power Company is performed. It is found that a proper dispatching schedule for the shunt capacitors and onload tap changers can be reached by the proposed method.
\end{abstract}

\section{Introduction}

In the operation of a distribution system, reactive power and voltage control is very important since system loss can be reduced and voltage profile can be improved through proper dispatch of reactive power control devices [1-9]. In general, there are three different types of reactive power control devices that are widely employed in a distribution system. An automatic voltage regulator (AVR) is usually installed to control the secondary bus voltage of a main transformer at a distribution substation through the action of transformer onload tap changers (OLTC). A shunt capacitor (SC) is installed at the low-voltage bus of the substation to adjust the reactive power flow through the main transformer. On each feeder, shunt capacitors may be installed to improve the voltage profile along the feeder. In the present work, control of reactive power/voltage is investigated using only OLTC and SC

\section{(C) IEE, 1995}

IEE Proceedings online no. 19952210

Paper first received 25th January 1995 and in revised form 3rd July 1995

The authors are with the Department of Electrical Engineering, National Taiwan University, Taipei, Taiwan, Republic of China at a distribution substation. Control of shunt capacitors on distribution feeders has been studied in previous works $[4,5]$.

The main purpose of this paper is to develop a new approach for reactive power/voltage control in a distribution substation which can overcome the disadvantages experienced in practical operation. The objective is to minimise reactive power flow over the main transformer and the transformer secondary bus voltage deviation subject to the constraints on secondary bus voltage limits and maximum allowable number of switching operations for the OLTC. To reach an optimal dispatch schedule for the SC and OLTC under the constraints in our study period (a day), the hourly real and reactive loads of the main transformer and its primary bus voltage are first forecast. Then a mathematical model is derived to ensure that secondary bus voltage limits are not violated when SC and OLTC are switched and to restrain the computational burden for the proposed dynamic programming approach.

To demonstrate the usefulness of the proposed method, dispatch of SC and OLTC for the main transformer at an automated distribution substation in Taipei, Taiwan is performed. It is found that the reactive power flow over the main transformer can be minimised and the secondary bus voltage can be kept very close to the specified value if the OLTC and SC are dispatched by using the proposed method. Practical constraints mentioned earlier are also satisfied.

\section{Description of study system}

The system under study is part of a $69 / 11.4 \mathrm{kV}$ distribution substation as shown in Fig. 1. The main transformer is equipped with an onload tap changer to keep its secondary bus voltage close to the preset value under changing load conditions. In addition, a shunt capacitor is installed at the $11.4 \mathrm{kV}$ secondary bus to compensate the reactive power flow through the main transformer. Based on TPC's operating policy, the shunt capacitor must be switched on/off according to system reactive power demand such that the reactive power flow over the main transformer is minimised. Thus, the power factor of the whole substation can be improved and the reactive power flows and losses on the subtransmission and transmission lines can also be reduced.

$\mathrm{Up}$ to now, automation for the most distribution substations within the service area of Taipei City District Office of Taiwan Power Company (TPC) has been completed. In normal operation, these unmanned, fully automated substations are monitored and controlled by 
the computers at the distribution dispatch and control centre (DDCC). As far as reactive power and voltage control at the distribution substation is concerned, the bus voltages, real and reactive powers of main transformers, on/off status of the SC, tap position of the OLTC, etc. are continuously monitored and recorded by the computer at DDCC through the action of remote terminal units (RTUs) and supervisory control and data acquisition system (SCADA) installed at the substation. Based on this information, which is stored in the database for $48 \mathrm{~h}$ and can be retrieved if necessary, it becomes possible to employ computer programs to determine whether the SC should be switched on/off or whether the tap position of the OLTC should be changed.

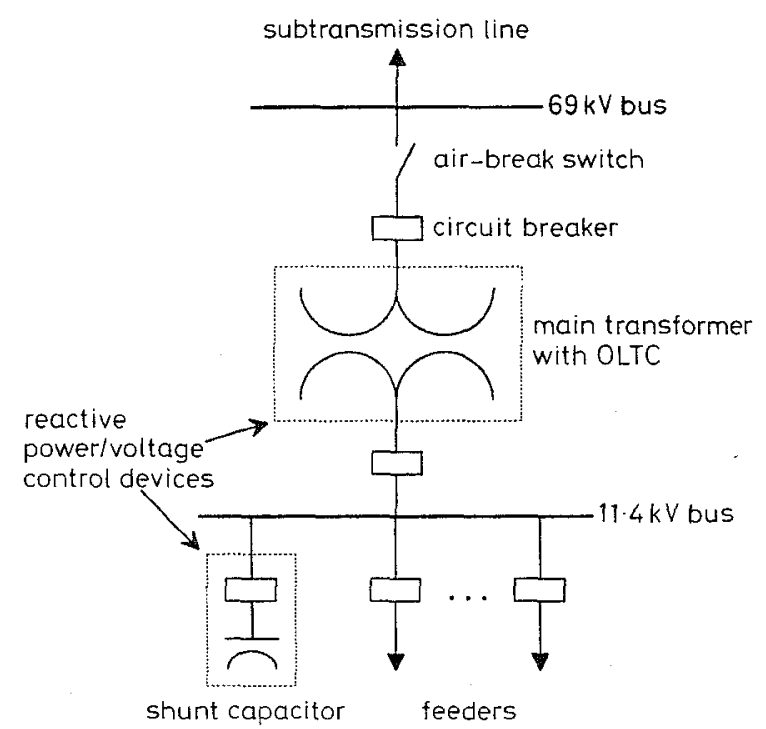

Fîg. 1 One-line diagram of part of $69 / 11.4 \mathrm{kV}$ distribution substation in Taipei

The reactive power/voltage control method currently used by TPC works well in most cases. However, it suffers from some disadvantages. For example, only realtime load data are employed to determine the dispatching strategy for the SC and OLTC. The unforeseen load changes in the near future may cause unnecessary, frequent switchings for the SC and OLTC. Improper co-ordination between the OLTC and SC may also be observed owing to the lack of rigorous mathematical models.

\section{Current practice of reactive power/voltage control in distribution substations at TPC}

At present, the reactive power/voltage control strategy in an automated distribution substation is shown in Fig. 2. There are three different control modes, i.e. the manual control mode (MANU mode), the automatic control mode (AUTO mode), and the reactive power device control mode (RPDC mode). In the manual control mode, the operators are directly in charge of the dispatch of SC and OLTC. Since it is not easy for inexperienced operators to set OLTC at proper tap position and switch on/off the SC at proper time, the MANU mode is employed only in very special situations where the operators' judgments and decisions in reactive power/voltage control are required. In the automatic control mode, the tap position of the OLTC is changed by the automatic voltage regulator which responds to the change in secondary bus voltage. On the other hand, the VA controller detects the reactive

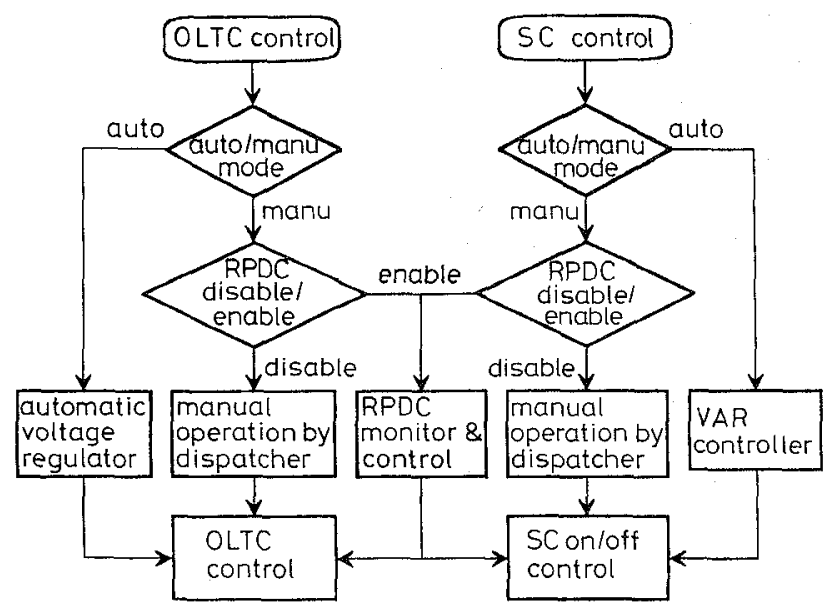

Fig.2 Flowchart of current reactive power/voltage control method at $T P C$

power flow over the main transformer and determines if it is necessary to switch on/off the SC. A few disadvantages of the automatic control mode are now described.

- Since the automatic voltage regulator and the VA controller function independently, good co-ordination between the OLTC control and SC on/off control is impossible.

- Some aged automatic voltage regulators may have delayed response to voltage deviations from the preset values. This may cause the OLTC fail to act at normal position when the SC circuit breaker is switched on/off or when there is severe load variation.

- Unnecessary frequent operation of the SC circuit breaker by the VA controller may be experienced in situations where there are temporary changes in the reactive power flow over the main transformer.

- The preset values of the VA controller must be reset manually following the change of SC installed capacities in different seasons. To co-ordinate the actions of automatic voltage regulator and the VA controller properly, the RPDC mode can be activated using computer software program. However, it still has some defects in practical operation.

(i) If the number of OLTC tap position movements in a day has exceeded the maximum allowable number of switching operations ( 30 in the present work), the RPDC mode will be disabled when it tries to activate further change in the tap position. The upper limit for tap position movement in a day has been set to avoid unnecessary OLTC actions and to avoid the reduction of life expectancy for OLTC owing to frequent switchings. Under this constraint, the OLTC tap position must be changed in most needful condition. In the current RPDC mode, the tap position of the OLTC is adjusted only based on present bus voltage. Therefore it may happen that the allowable thirty switching operations are exhausted in the afternoon and it is impossible to make any tap adjustment in the future hours.

(ii) In current RPDC mode of operation, the minimum on time and minimum off time for the SC are set to be 20 and 5 min, respectively. This is done to avoid too frequent switching operations of the SC. However, when the reactive power flow over the main transformer is very close to $50 \%$ of the rated capacity of the $\mathrm{SC}$, frequent switching operations are still observed for the $\mathrm{SC}$ in this mode. 
(iii) In the RPDC mode, secondary bus voltage change due to the switching of $\mathrm{SC}$ is estimated by using the following equation:

$\Delta V($ in $\mathrm{kV})=0.23 \times(Q$ value of the $\mathrm{SC}($ in $\mathrm{MVAr})) / 6$

This is a very rough formula. In fact, the voltage deviation resulting from capacitor switching will be a function of the real and reactive power load demand. A mathematical formula is given in Section 4 to estimate secondary bus voltages in a more accurate manner.

(iv) In the RPDC mode, the $\mathrm{SC}$ is switched on/off when the lagging/leading reactive power flow over the main transformer exceeds $50 \%$ of the rated SC capacity. Before the SC circuit breaker is operated, the secondary bus voltage after the $\mathrm{SC}$ is switched is first estimated to ensure that the bus voltage will remain within the allowable limit ( $\pm 5 \%$ of the objective value). The SC is switched only when the SC switching will not cause voltage limit violations. This is a major advantage of the RPDC mode which can not be achieved by the AUTO mode. However, in situations where the voltage limit violations are caused by improper OLTC tap positions, the SC switching operation will be inhibited in this mode to prevent bus voltage violations. Reactive power control is not carried out in this case. This motivates the development of a more rigorous control method, described in Section 5.

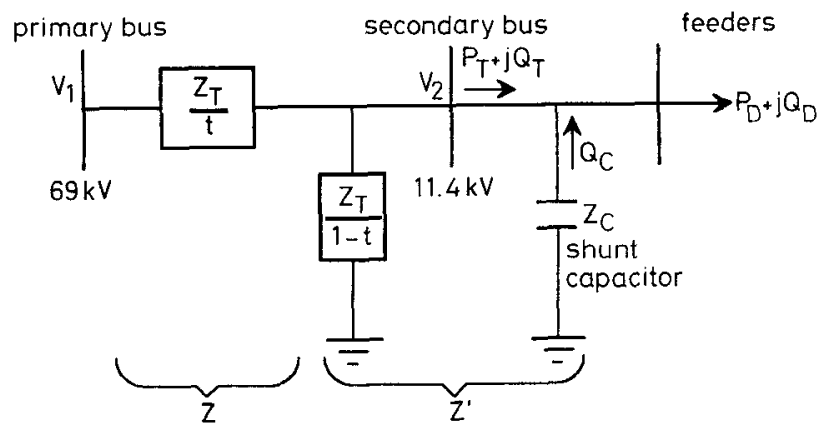

Fig.3 Mathematical model of main transformer with OLTC and bus shunt capacitor

In summary, all of those disadvantages that happen to current practice of reactive power/voltage control in distribution substation at TPC may be avoided by the proposed approach.

\section{Bus voltage estimation formula}

To estimate main transformer secondary bus voltage, the mathematical model for the system in Fig. 1 is depicted in Fig. 3. The following symbols have been used in Fig. 3:

$V_{1}=$ main transformer primary bus voltage $(69 \mathrm{kV})$

$V_{2}=$ main transformer secondary bus voltage $(11.4 \mathrm{kV})$

$Z_{T}=$ main transformer impedance

$t=$ transformer voltage ratio

$P_{D}=$ real power demand

$Q_{D}=$ reactive power demand

The main transformer resistance and its magnetising admittance are neglected in the present study. To find the secondary bus voltage $\left|V_{2}\right|$, let us first write down the real power demand and reactive power demand equations:

$$
P_{D}=\frac{\left|V_{1}\right|\left|V_{2}\right|}{|Z|} \sin \left(\angle V_{1}-\angle V_{2}\right)=\frac{\left|V_{1}\right|\left|V_{2}\right|}{|Z|} \sin \theta_{12}
$$

$O_{D}=\left(-\frac{\left|V_{2}\right|^{2}}{|Z|}+\frac{\left|V_{1}\right|\left|V_{2}\right|}{|Z|} \cos \theta_{12}\right)-\frac{\left|V_{2}\right|^{2}}{\left|Z^{\prime}\right|}$

Eqn. 2 is rewritten as

$$
\left[Q_{D}+\frac{\left|V_{2}\right|^{2}}{|Z|}+\frac{\left|V_{2}\right|^{2}}{\left|Z^{\prime}\right|}\right]^{2}=\frac{\left|V_{1}\right|^{2}\left|V_{2}\right|^{2}}{|Z|^{2}} \cos ^{2} \theta_{12}
$$

By combining eqns. 1 and 3 ,

where

$$
\left[Q_{D}+\frac{\left|V_{2}\right|^{2}}{\left|Z^{\prime \prime}\right|}\right]^{2}=\frac{\left|V_{1}\right|^{2}\left|V_{2}\right|^{2}}{|Z|^{2}}-P_{D}^{2}
$$

Expanding eqn. 4 gives

$$
\left|Z^{\prime \prime}\right|=\left[\frac{1}{|Z|}+\frac{1}{\left|Z^{\prime}\right|}\right]^{-1}
$$

$$
\begin{aligned}
& \left|V_{2}\right|^{4}+\left(2 Q_{D}\left|Z^{\prime \prime}\right|-\frac{\left|Z^{\prime \prime}\right|^{2}}{|Z|^{2}}\left|V_{1}\right|^{2}\right)\left|V_{2}\right|^{2}+ \\
& \left(P_{D}^{2}+Q_{D}^{2}\right)\left|Z^{\prime \prime}\right|^{2}=0
\end{aligned}
$$

From eqn. 5 , the secondary bus voltage magnitudes $\left|V_{2}\right|$ is expressed as

$$
\begin{aligned}
\left.V_{2}\right|^{2}= & \frac{1}{2} \frac{\left|Z^{\prime \prime}\right|^{2}}{|Z|^{2}}\left|V_{1}\right|^{2}-Q_{D}\left|Z^{\prime \prime}\right| \pm \\
& {\left[\frac{1}{4} \frac{\left|Z^{\prime \prime}\right|^{4}}{|Z|^{4}}\left|V_{1}\right|^{4}-P_{D}^{2}\left|Z^{\prime \prime \prime}\right|^{2}-Q_{D} \frac{\left|Z^{\prime \prime}\right|^{3}}{|Z|^{2}}\left|V_{1}\right|^{2}\right]^{1 / 2} }
\end{aligned}
$$

Now consider the situation where the shunt capacitor is switched off. In this case, $Z^{\prime}=Z_{T} /(1-t)$ and

$$
\begin{aligned}
& \left|Z^{\prime \prime}\right|=\left(\frac{1}{|Z|}+\frac{1}{\left|Z^{\prime}\right|}\right)^{-1}=\left(\frac{t}{\left|Z_{T}\right|}+\frac{1-t}{\left|Z_{T}\right|}\right)^{-1}=\left|Z_{T}\right| \\
& \frac{\left|Z^{\prime \prime}\right|}{|Z|}=\left|Z_{T}\right| /\left(\left|Z_{T}\right| / t\right)=t
\end{aligned}
$$

By using eqns. 7 and 8 , the transformer voltage ratio $t$ can be derived from eqn. 4 and eqn. 6 can be simplified as follows:

$$
\begin{aligned}
t= & \left\{\frac{\left|Z_{T}\right|^{2}}{\left|V_{1}\right|^{2}\left|V_{2}\right|^{2}}\left[\left(Q_{D}+\frac{\left|V_{2}\right|^{2}}{\left|Z_{T}\right|}\right)^{2}+P_{D}^{2}\right]\right\}^{1 / 2} \\
\left|V_{2}\right|^{2}= & \frac{1}{2} t^{2}\left|V_{1}\right|^{2}-\left|Z_{T}\right| Q_{D}+ \\
& {\left[\frac{1}{4} t^{4}\left|V_{1}\right|^{4}-\left|Z_{T}\right|^{2} P_{D}^{2}-t^{2}\left|Z_{T}\right| Q_{D}\left|V_{1}\right|^{2}\right]^{1 / 2} }
\end{aligned}
$$

One of the objectives in reactive power/voltage control is to keep secondary bus voltage $\left|V_{2}\right|$ as close to $1.0 \mathrm{p}$.u. $(6.73 \mathrm{kV}$ per phase) as possible no matter how the load $\left(P_{D}+j Q_{D}\right)$ changes with time. In our study, the ideal transformer voltage ratio is first found out by substituting $\left|V_{2}\right|=1.0$ p.u. into eqn. 9. In practice, the transformer voltage ratio can only be changed by multiples of $0.0125 \mathrm{p} . \mathrm{u}$. Thus, the actual transformer voltage ratio must be rounded off to the nearest multiple of $0.0125 \mathrm{p} . \mathrm{u}$. When this is done, the actual transformer voltage ratio $t$ is related to the OLTC tap position $(T A P)$ as follows

$$
t=1+T A P(0.0125)
$$

Note that the OLTC tap position TAP should be an integer. With the actual transformer voltage ratio at hand, the secondary bus voltage that we want to control can be derived by using eqn. 10 .

When the shunt capacitor is switched on, the impedance $Z^{\prime}$ becomes

$$
Z^{\prime}=\left[\left(\left|Z_{T}\right| /(1-t)\right)^{-1}-\left(Z_{C}\right)^{-1}\right]^{-1}
$$


In this case,

$$
\begin{aligned}
& \left|Z^{\prime \prime}\right|=k\left|Z_{T}\right| \\
& \frac{\left|Z^{\prime \prime}\right|}{|Z|}=k t
\end{aligned}
$$

where

$$
k=\frac{\left|Z_{C}\right|}{\left|Z_{C}\right|-\left|Z_{T}\right|}
$$

Eqns. 9 and 10 are now replaced by eqns. 15 and 16, respectively:

$$
\begin{aligned}
& t=\left\{\frac{\left|Z_{T}\right|^{2}}{\left|V_{1}\right|^{2}\left|V_{2}\right|^{2}}\left[\left(Q_{D}+\frac{\left|V_{2}\right|^{2}}{k\left|Z_{T}\right|}\right)^{2}+P_{D}^{2}\right]\right\}^{1 / 2} \\
& \left|V_{2}\right|^{2}=\frac{1}{2}(k t)^{2}\left|V_{1}\right|^{2}-\left(k\left|Z_{T}\right|\right) Q_{D}+ \\
& \quad\left[\frac{1}{4}(k t)^{4}\left|V_{1}\right|^{4}-\left(k\left|Z_{T}\right|\right)^{2} P_{D}^{2}-(k t)^{2}\left(k\left|Z_{T}\right|\right) Q_{D}\left|V_{1}\right|^{2}\right]^{1 / 2}
\end{aligned}
$$

In this paper it is reasonable to assume that $K=1$ when the shunt capacitor is switched off.

\section{Dynamic programming approach for reactive power/voltage control}

Before the proposed dynamic programming (DP) approach for reactive power/voltage control is described, the hourly real and reactive loads of a main transformer and its primary bus voltage are first forecast.

In the present work, the method in $[10,11]$ is adopted to predict the hourly loads of a main transformer in a day. On the other hand, the estimation error of the primary bus voltage will affect the results slightly for our problem since it is about ten times the secondary bus voltage (phase voltage). So it is forecast as the average value of the actual primary bus voltage in the past month. In fact, it is found from the results in Section 6 that the primary bus voltages estimated in this manner are very close to actual primary bus voltages.

To describe the proposed DP approach, let us define

$$
\begin{aligned}
& X_{i}=1, \mathrm{SC} \text { is on at hour } i \\
& X_{i}=0, \mathrm{SC} \text { is off at hour } i
\end{aligned}
$$

It has been mentioned in Section 4 that the relationship between the transformer secondary bus voltage $\left|V_{2}\right|$ and transformer voltage ratio $t$ is expressed in eqns. 10 and 16 for the case where the SC is off $\left(X_{i}=0\right)$ and the case where SC is on $\left(X_{i}=1\right)$. With the hourly forecast data $\left(P_{D i}, Q_{D i}\right.$, and $\left.\left|V_{1 i}\right|\right)$ and the secondary bus voltage estimation formula (eqns. 10 and 16) at hand, we proceed to determine an optimal reactive power/voltage control strategy for the main transformer at the distribution substation. In other words, we require a set of control variables $X_{i}(i=1, \ldots, 24)$ for the $\mathrm{SC}$ and $T A P_{i}(i=1$, ..., 24) for the OLTC such that the reactive power flow over the main transformer is kept as small as possible and the secondary bus voltage is maintained as close to the specified value (1.0p.u.) as possible at all hours in a day. Practical constrains on secondary bus voltage limits and maximum allowable number of switching operations in a day for the OLTC are taken into account.

In the mathematical expressions, the reactive power/ voltage control problem can be stated as follows.
Minimise

$$
\begin{gathered}
J=\sum_{i=1}^{24}\left(\left|V_{2 s p e c}\right|-\left|V_{2 i, a c t}\right|\right)+\sum_{i=1}^{24}\left|Q_{T i}\right| \\
= \\
\begin{array}{l}
\text { subject to } \\
\sum_{1}^{24}\left|T A P_{i}-T A P_{i-1}\right| \leq 30
\end{array} \\
\sum_{i=2} \mid J_{2} \\
\left|V_{2}^{\text {min }}\right| \leq\left|V_{2 i, a c t}\right| \leq\left|V_{2}^{\text {max }}\right| \quad i=1, \ldots, 24
\end{gathered}
$$

where

$\left|V_{2 \text { spec }}\right|=$ specified secondary bus voltage (1.0p.u.)

$\left|V_{2 i, a c t}\right|=$ actual secondary bus voltage at hour $i$

$Q_{T i}=$ main transformer reactive power flow at hour $i$

$T A P_{i}=$ OLTC tap position at hour $i$

$\left|V_{2}^{\text {min }}\right|=$ secondary bus voltage lower limit (0.95p.u.)

$$
\left|V_{2}^{\max }\right|=\text { secondary bus voltage upper limit }
$$
(1.05 p.u.)

Note that the objective function $J$ as defined in eqn. 17 indicates that we want to control secondary bus voltage $\left|V_{2}\right|$ and transformer reactive power flow $\left|Q_{T}\right|$ at the same time. The constraints on the maximum allowable number of switching operations for the OLTC and the secondary bus voltage limit are expressed in eqns. 18 and 19 , respectively.

To find the control variables $X_{i}(i=1, \ldots, 24)$ for the $\mathrm{SC}$ and $T A P_{i}(i=1, \ldots, 24)$ for the OLTC which minimise the objective function $J$ in eqn. 17 subject to constraints in eqns. 18 and 19, DP approach is utilised. Fig. 4 depicts the state diagram and search paths for the DP approach.

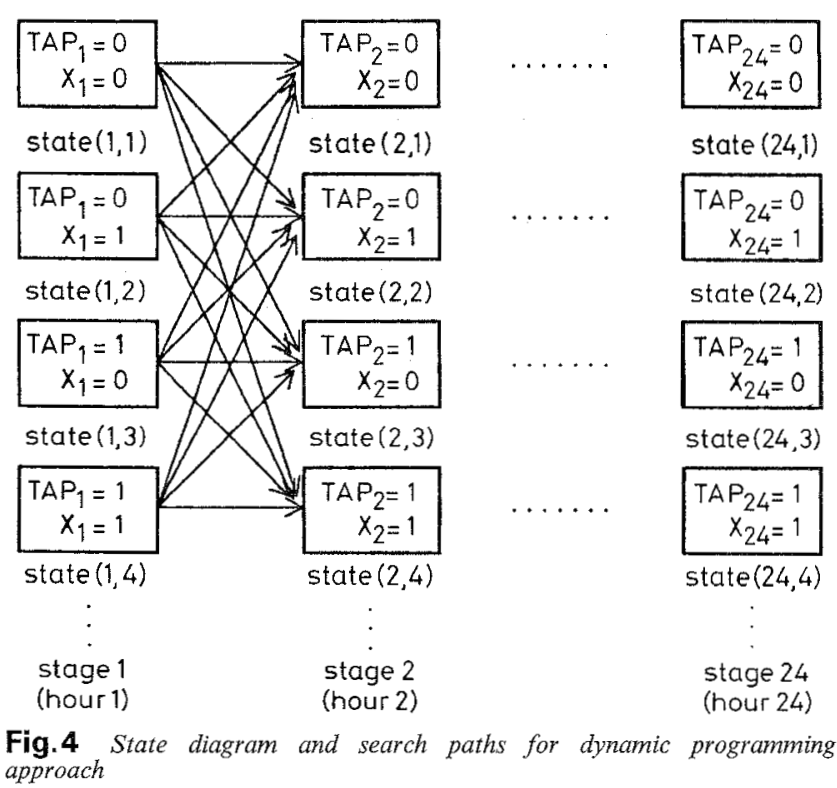

Since there are two possible values for $X_{i}\left(X_{i}=1\right.$ or $0)$ and there are 17 possible values for $T_{A} P_{i}\left(T A P_{i}=-\right.$ $8,-7,-6, \ldots, 0,1,2, \ldots, 8)$, there will be $34(=2 \times 17)$ states at each stage. Therefore, we need to traverse and save $34^{24}\left(\simeq 5.69 \times 10^{36}\right)$ possible search paths through the system states at 24 stages. Our purpose is to find one feasible path that satisfies the constraints described in eqns. 18 and 19 , and gives the least objective function $J$ over the study period. If we try to solve the problem using the state diagram and search paths in 
Fig. 4, we face the difficulty of too much computer memory and intensive computational effort.

To reduce the computational burden, the number of states at each stage and the search paths must be reduced. In the present work, we first find the optimal tap position $T A P_{i \text { optimal }}$ that will make $\left|V_{2}\right|$ closest to objective value at each stage. Only the three tap positions which are closest to $T A P_{i \text { optimal }}$ are saved at each stage. In this way, there are two possible values for $X_{i}$ and only three possible values for $T A P_{i}$. So each state node at a given stage will create only six state nodes at the next stage. To further improve the efficiency of the DP approach, the maximum size of the state space must be limited. To do this, we restrict the number of states from stage 2 to stage 24 to 36 states. In other words, only those 36 states with the lowest objective values among the $216(=36 \times 6)$ states are stored. Since there are six states at the first stage, there will be $6+36 \times 23=834$ states in all. It means that the nearoptimal solution can be reached at minimal computational effort.

\section{Example}

To demonstrate the usefulness of the proposed method, reactive power/voltage control is performed on a main transformer at CHENG-CHUNG distribution substation in Taipei, Taiwan. The one-line diagram is shown in Fig. 1. The main transformer rating MVA and its impedance $\left(Z_{T}\right)$ are 33 and 0.1223 p.u., respectively. The installed capacity for SC is $8.4 \mathrm{MVAr}$. Due to limited space in this paper, only one example is given to demonstrate the results from load forecasting and reactive power/voltage control for the case of weekdays.

Reactive power/voltage control for 22nd June 1994 (Wednesday): Table 1 gives the results from load forecasting for 22 nd June 1994. As mentioned earlier, the hourly primary bus voltage is forecast as the average value of the actual primary bus voltage in the past month.

With the forecast data, we can proceed to use the DP approach in Section 5 to determine the optimal dispatch schedule for the SC and OLTC. In the present work, the desired main transformer secondary bus voltage has been set as current dispatch value $(6.73 \mathrm{kV}$ per phase or 1.0 p.u.) at DDCC for each hour.

The resulting reactive power/voltage dispatch schedule from the proposed approach and the historical dispatch schedule at DDCC of TPC are summarised in Table 2. It is observed that better control on secondary bus voltage can be achieved by the proposed approach than the current control method.

\section{Discussion}

In this paper, equal weights are put on voltage control and reactive power control. If it is desired to put more emphasis on voltage control or reactive power control, one can use different weighting factors for functions $J_{1}$ and $J_{2}$.

At present, reactors are installed at EHV $(345 \mathrm{kV})$ substations while capacitors are installed at primary $(161 \mathrm{kV})$ substations and distribution substations at

Table 1: Load forecast results for 22nd June 1994

\begin{tabular}{|c|c|c|c|c|c|c|c|c|}
\hline hour & $\begin{array}{l}P_{\text {act }} \\
\text { (MW) }\end{array}$ & $\begin{array}{l}P_{\text {For }} \\
(\mathrm{MW})\end{array}$ & $\begin{array}{l}\left|P_{a c t}-P_{\text {Forl }}\right| \\
\text { (MW) }\end{array}$ & $\%$ error & $\begin{array}{l}Q_{B C t} \\
\text { (MVAr) }\end{array}$ & $\begin{array}{l}Q_{\text {For }} \\
\text { (MVAr) }\end{array}$ & $\begin{array}{l}\left|Q_{a c t}-Q_{\text {For }}\right| \\
\text { (MVAr) }\end{array}$ & $\%$ error \\
\hline 1 & 5.376 & 5.269 & 0.107 & 0.461 & -1.414 & -1.517 & 0.103 & 1.237 \\
\hline 2 & 4.955 & 4.862 & 0.093 & 0.398 & -1.608 & -1.679 & 0.071 & 0.852 \\
\hline 3 & 4.714 & 4.566 & 0.148 & 0.638 & -1.753 & -1.779 & 0.026 & 0.306 \\
\hline 4 & 4.482 & 4.387 & 0.095 & 0.408 & -1.877 & -1.859 & 0.018 & 0.221 \\
\hline 5 & 4.394 & 4.285 & 0.109 & 0.471 & -1.845 & -1.933 & 0.088 & 1.053 \\
\hline 6 & 4.407 & 4.341 & 0.066 & 0.282 & -1.806 & -1.834 & 0.028 & 0.335 \\
\hline 7 & 5.872 & 5.825 & 0.047 & 0.202 & -1.040 & -1.052 & 0.012 & 0.144 \\
\hline 8 & 9.639 & 9.259 & 0.380 & 1.633 & 0.975 & 0.928 & 0.047 & 0.557 \\
\hline 9 & 16.997 & 16.444 & 0.553 & 2.379 & 5.113 & 4.857 & 0.256 & 3.068 \\
\hline 10 & 20.446 & 20.301 & 0.145 & 0.624 & 6.980 & 6.999 & 0.019 & 0.223 \\
\hline 11 & 22.377 & 22.283 & 0.094 & 0.403 & 8.024 & 8.044 & 0.020 & 0.240 \\
\hline 12 & 23.266 & 23.380 & 0.114 & 0.492 & 8.355 & 8.485 & 0.130 & 1.558 \\
\hline 13 & 22.777 & 22.800 & 0.023 & 0.098 & 8.160 & 8.164 & 0.004 & 0.044 \\
\hline 14 & 22.495 & 22.439 & 0.056 & 0.241 & 7.842 & 7.873 & 0.031 & 0.377 \\
\hline 15 & 23.126 & 22.875 & 0.251 & 1.080 & 8.055 & 8.262 & 0.207 & 2.480 \\
\hline 16 & 23.097 & 22.650 & 0.447 & 1.921 & 8.124 & 8.288 & 0.164 & 1.958 \\
\hline 17 & 22.511 & 22.021 & 0.490 & 2.108 & 8.156 & 8.181 & 0.025 & 0.294 \\
\hline 18 & 18.872 & 18.950 & 0.078 & 0.336 & 6.617 & 6.781 & 0.164 & 1.968 \\
\hline 19 & 16.344 & 16.570 & 0.226 & 0.971 & 5.125 & 5.539 & 0.414 & 4.957 \\
\hline 20 & 14.636 & 14.863 & 0.227 & 0.976 & 3.992 & 4.446 & 0.454 & 5.430 \\
\hline 21 & 13.429 & 13.371 & 0.058 & 0.250 & 3.461 & 3.634 & 0.173 & 2.070 \\
\hline 22 & 10.462 & 10.163 & 0.299 & 1.284 & 1.566 & 1.599 & 0.033 & 0.394 \\
\hline 23 & 7.532 & 7.308 & 0.224 & 0.961 & -0.349 & -0.290 & 0.059 & 0.709 \\
\hline 24 & 6.405 & 6.153 & 0.252 & 1.084 & -1.138 & -1.129 & 0.009 & 0.107 \\
\hline
\end{tabular}


Table 2: Reactive power/voltage dispatch schedule from proposed approach and current dispatch method at DDCC of TPC (22nd June 1994)

\begin{tabular}{|c|c|c|c|c|c|c|c|c|c|c|c|c|c|c|}
\hline \multirow{2}{*}{ hour } & \multicolumn{2}{|c|}{$T A P_{i}$} & \multicolumn{2}{|l|}{$X_{i}$} & \multicolumn{2}{|l|}{$V_{1}$} & \multicolumn{2}{|l|}{$V_{2}$} & \multicolumn{2}{|l|}{$J$} & \multicolumn{2}{|l|}{$J_{1}$} & \multicolumn{2}{|l|}{$J_{2}$} \\
\hline & $P$ & C & $P$ & C & $\mathrm{P}$ & C & $P$ & C & $\mathrm{P}$ & C & $P$ & C & $\mathrm{P}^{*}$ & $\mathrm{C}^{*}$ \\
\hline 1 & 0 & -3 & 0 & 0 & 0.982 & 0.978 & 1.012 & 0.970 & 0.057 & 0.072 & 0.012 & 0.030 & 0.045 & 0.042 \\
\hline 2 & -1 & -3 & 0 & 0 & 0.984 & 0.980 & 0.990 & 0.973 & 0.118 & 0.147 & 0.022 & 0.057 & 0.096 & 0.090 \\
\hline 3 & -1 & -3 & 0 & 0 & 0.987 & 0.981 & 0.993 & 0.975 & 0.178 & 0.225 & 0.029 & 0.082 & 0.149 & .143 \\
\hline 4 & -1 & -3 & 0 & 0 & 0.988 & 0.984 & 0.994 & 0.976 & 0.239 & 0.305 & 0.035 & 0.106 & 0.204 & 0.199 \\
\hline 5 & -1 & -3 & 0 & 0 & 0.987 & 0.990 & 0.994 & 0.984 & 0.303 & 0.377 & 0.041 & 0.122 & 0.262 & 0.255 \\
\hline 6 & -1 & -3 & 0 & 0 & 0.988 & 0.980 & 0.994 & 0.972 & 0.364 & 0.459 & 0.047 & 0.150 & 0.317 & 0.309 \\
\hline 7 & 0 & -3 & 0 & 0 & 0.977 & 0.971 & 1.006 & 0.958 & 0.401 & 0.532 & 0.053 & 0.192 & 0.348 & 0.340 \\
\hline 8 & 0 & -3 & 0 & 0 & 0.989 & 0.997 & 1.010 & 0.970 & 0.439 & 0.591 & 0.063 & 0.222 & 0.376 & 0.369 \\
\hline 9 & -3 & -4 & 1 & 1 & 1.004 & 0.997 & 1.000 & 0.981 & 0.533 & 0.687 & 0.063 & 0.241 & 0.470 & 0.446 \\
\hline 10 & -2 & -4 & 1 & 1 & 1.006 & 0.996 & 1.001 & 0.975 & 0.564 & 0.731 & 0.064 & 0.266 & 0.500 & 0.465 \\
\hline 11 & -1 & -4 & 1 & 1 & 1.006 & 1.006 & 1.002 & 0.982 & 0.567 & 0.758 & 0.066 & 0.284 & 0.501 & 0.474 \\
\hline 12 & -1 & -4 & 1 & 1 & 1.008 & 1.016 & 1.002 & 0.990 & 0.582 & 0.785 & 0.068 & 0.294 & 0.514 & 0.491 \\
\hline 13 & -2 & -4 & 1 & 1 & 1.010 & 1.015 & 1.000 & 0.994 & 0.588 & 0.798 & 0.068 & 0.300 & 0.520 & 0.498 \\
\hline 14 & -1 & -4 & 1 & 1 & 1.004 & 1.001 & 1.001 & 0.978 & 0.593 & 0.827 & 0.069 & 0.322 & 0.524 & 0.505 \\
\hline 15 & -2 & -4 & 1 & 1 & 1.010 & 1.006 & 0.999 & 0.981 & 0.602 & 0.857 & 0.070 & 0.341 & 0.532 & 0.516 \\
\hline 16 & -2 & -4 & 1 & 1 & 1.011 & 1.009 & 1.001 & 0.984 & 0.612 & 0.885 & 0.071 & 0.357 & 0.541 & 0.528 \\
\hline 17 & -3 & -4 & 1 & 1 & 1.016 & 1.020 & 0.998 & 0.999 & 0.620 & 0.892 & 0.073 & 0.358 & 0.547 & 0.534 \\
\hline 18 & -4 & -6 & 1 & 1 & 1.017 & 1.015 & 1.001 & 0.988 & 0.658 & 0.940 & 0.074 & 0.370 & 0.584 & 0.570 \\
\hline 19 & -4 & -6 & 1 & 1 & 1.009 & 1.006 & 0.998 & 0.985 & 0.733 & 1.034 & 0.076 & 0.385 & 0.657 & 0.649 \\
\hline 20 & -5 & -6 & 1 & 1 & 1.012 & 1.009 & 0.998 & 0.988 & 0.841 & 1.160 & 0.078 & 0.397 & 0.763 & 0.763 \\
\hline 21 & -1 & -7 & 0 & 1 & 1.013 & 1.017 & 0.997 & 0.994 & 0.953 & 1.299 & 0.081 & 0.403 & 0.872 & 0.896 \\
\hline 22 & 0 & -7 & 0 & 0 & 0.991 & 0.997 & 1.009 & 0.957 & 1.010 & 1.389 & 0.090 & 0.446 & 0.920 & 0.943 \\
\hline 23 & 0 & -6 & 0 & 0 & 0.982 & 0.984 & 1.008 & 0.955 & 1.026 & 1.444 & 0.098 & 0.491 & 0.928 & 0.953 \\
\hline 24 & 0 & -3 & 0 & 0 & 0.981 & 0.968 & 1.010 & 0.960 & 1.070 & 1.518 & 0.108 & 0.531 & 0.962 & 0.987 \\
\hline
\end{tabular}

Taiwan Power Company. Thus, reactors are not considered in the present work because we are examining reactive power/voltage control problem in a distribution substation. However, it is easy to incorporate reactance into the mathematical model in Section 4.

In real system operation, it may happen that system load varies to a great extent within $1 \mathrm{~h}$. In addition, certain emergency conditions may cause load shedding or load transfer. In this case, the preset tap position may be adjusted using the monitored voltage, load data and eqns. 9 and 11 .

\section{Conclusions}

In this paper, reactive power/voltage control in a distribution substation has been studied. The purpose was to find a proper dispatching schedule for the shunt capacitor and onload tap changer such that main transformer secondary bus voltage can be maintained close to the specified value and the reactive power flow over the main transformer can be kept minimal. To reach the desired dispatching schedule, the real and reactive power demands of a main transformer and its primary bus voltage for the next $24 \mathrm{~h}$ were first forecast. With these data at hand, a mathematical model was built to estimate transformer secondary bus voltage as a function of the control variables, OLTC tap positions and the on/off status of the shunt capacitor. An approach based on dynamic programming has been presented to reach the desired reactive power/voltage dispatch schedule. From the results, the bus voltage can be controlled very close to the specified value via the action of OLTC while the reactive power flow over the main transformer can be kept minimal through the switching operations of the SC.

\section{Acknowledgments}

The authors express their gratitude to C.F. Song, T.M. Cheng, Y.T. Wang, L.C. Lin, N.C. Yen, K.H. Chang, C.Y. Wang and H.L. Cheng of TPC for providing the valuable system data and dispatch experience. Financial support was given to this work by the National Science Council of ROC under contract number NSC83-0404-E002-067.

\section{References}

1 GÖNEN,T.: 'Electric power distribution system engineering' (McGraw-Hill, 1986)

2 BRIDENBAUGH,C.J., DIMASCIO,D.A., and D'AGUILA,R.: 'Voltage control improvement through capacitor and transformer tap optimisation', IEEE Trans., 1992, PWRS-7, pp. 222-227

3 HSU,Y.Y., HO, K.L., LIANG,C.C., LAI,T.S., CHEN, K.K. and CHANG, B.S.: "Voltage control using a combined integer linear programming and rule-base approach', IEEE Trans., 1992, PWRS-7, pp. 744-752 
4 HSU, Y.Y., and YANG, C.C.: 'A hybrid artificial neural networkdynamic programming approach for feeder capacitor scheduling', Paper 93 SM 505-8, IEEE/PES 1993 Summer Meeting

5 HSU, Y.Y., and KUO, H.C.: 'Dispatch of capacitors on distribution system using dynamic programming', IEE Proc. C, 1993, 139, pp. $433-438$

6 GRAINGER,J.J., CIVANLAR,S., and LEE,S.H.: 'Optimal design and control scheme for continuous capacitive compensation of distribution feeders', IEEE Trans., 1983, PAS-102, pp. 3271-3278

7 GRAINGER, J.J., and CIVANLAR, S.: 'Volt/Var control on distribution systems with lateral branches using shunt capacitors and voltage regulators', IEEE Trans., 1985, PAS-104, pp. 3278-3297
8 KAPLAN, M: 'Optimisation of number, location, size, control type, and control settings of shunt capacitors on radial distribution feeders', IEEE Trans., 1984, PAS-102, pp. 2659-2663

9 SANTOSO,N.I., and TAN,O.T.: 'Neural-net based real-time control of capacitors installed on distribution systems', IEEE Trans., 1990, PWRD-5, pp. 266-272

10 GROSS,G., and GALIANA,F.D.: 'Short term load forecasting', Proc. IEEE, 1987, 75, (12), pp. 1558-1573

11 HO, K.L., HSU, Y.Y., LIANG,C.C., LAI, T.S., and CHEN, K.K.: 'Short term load forecasting of Taiwan power system using a knowledge-based expert system', IEEE Trans., 1990, PWRS-5, pp. 1214-1221 